\title{
The Dynamical Entropy
}

\author{
V. M. Somsikov ${ }^{1}$
}

${ }^{1}$ Institute of Ionosphere, Almaty, Kazakhstan, 050020

\begin{abstract}
The concept of dynamic entropy (D-entropy), proposed in the mechanics of structured particles is discussed. D-entropy is defined as the relative increase in the internal energy of the system due to its motion energy in the inhomogeneous field of external force. D-entropy is determined completely from the motion equation of the system without any statistical laws. Comparison of D-entropy with thermodynamic entropy of Clausius, Boltzmann's entropy and Kolmogorov - Sinai entropy is performed. The numerical results calculations of the changes of the systems' D-entropy consisting from the different numbers of elements during their motions into the non-homogeneous space are given. Areas of application D- entropy and the possibility of its use for the analysis of dynamic systems are discussed.
\end{abstract}

Keywords: dynamics, entropy, irreversibility, classical mechanics.

\section{Introduction}

Entropy is one of the most important parameters characterizing the substance. Arisen concept of entropy in physics is used in practically in all fields of science, which indicates its fundamental importance [1]. But up to now, the problem of a clear physical meaning of entropy exists. Solution of this problem rests against the need to find the connection between entropy and dynamic properties of various systems.

In the modern form the concept of entropy appeared empirically in thermodynamics as a function of the state of matter. This means that each state of matter associated with a certain amount of entropy, which characterizes this state. The very concept of entropy in thermodynamics was introduced by the German physicist R. Clausius in 1865 . He defined it as a relative increase in the heat of the system $[1,2]$ :

$d S^{c}=d Q /\left.T\right|_{N \rightarrow \infty}$

Here $S^{c}$ - Clausius entropy, $Q$ - heart, $T$ temperature of the substance, $N$ - the number of particles.

Entropy as well as energy is additive quantity. If deviations from thermodynamic equilibrium are small and the condition of local thermodynamic equilibrium have a place, the entropy of system is put into correspondence to a given substance and it is the sum of the entropies of its equilibrium parts [1,2, and 9]. It was found that the change in entropy of matter obeys to the second law of thermodynamics, according to which it can only increase, that is, $d S^{c} / d t \geq 0 \quad$ [1]. The second law of thermodynamics was obtained empirically.

Deeply supporter of atomic-molecular theory of the structure of matter, Boltzmann was the first who tried to justify the second law of thermodynamics in the framework of the laws of classical mechanics. Within the framework of molecular - kinetic theory he proved $\mathrm{H}$ - theorem. According to this theorem the entropy of the substance from interacting atoms or molecules should increase. But the proof of the $\mathrm{H}$ theorem based on the statistical laws. Therefore, as indicated by Poincare, the proof is not sufficiently justified [4]. Moreover, Poincaré showed that the conclusions $\mathrm{H}$ - theorem contradict to the formalism of classical mechanics, according to which the system must be reversible. By the way, this is a contradiction between physics and the real nature was the first from three major problems facing the physics [5]. However, as experience has shown, $\mathrm{H}$ - theorem is well satisfied for real systems [2].

Using statistical laws within the molecular-kinetic theory, the L. Boltzmann has found expression for the entropy, which is determined by the number of microstates that implement the corresponding macrostate of the system $[1,2]$ :

$S^{B}=\kappa \ln W \quad(2)$

Here $S^{B}$ - Boltzmann entropy, $\kappa$ - Boltzmann constant, $W$ - the number of microstates that implements this state of matter.

This article is published under the terms of the Creative Commons Attribution License 4.0

Author(s) retain the copyright of this article. Publication rights with Alkhaer Publications.

Published at: http://www.ijsciences.com/pub/issue/2015-05/

Article Number: V4201505712; Online ISSN: 2305-3925; Print ISSN: 2410-4477 
Ideas that used in obtaining Boltzmann formula (2), in particular, the hypothesis about equal probability of impact parameters, the hypothesis about equivalence the averaging over time to the averaging in space, served as a starting points for a very important in the statistical physics of the ergodic theory $[6,7]$. It is in connection with the formula (2) with time under the concept of entropy begun to understand the measure of system's disorder.

In general, the empirical formula (1) and the statistical formula (2) are not associated with the dynamic characteristics of the system elements. That is, the relationship between dynamic parameters of systems and probabilistic hypotheses that form the basis of evidence of the formula (2) remains unresolved problem and requires proof. But it is precisely the dynamic processes associated with the movements of molecules of matter, are the basis of all evolutionary processes that determine its states.

A very serious step towards solving the problem of substantiation thermodynamics, dynamics, and theory of dynamic chaos in the framework of the laws of classical mechanics should be considered of the formula of entropy obtained by Sinai [6-9]:

$$
S^{K S}=\lim _{d(0) \rightarrow 0, t \rightarrow \infty} \frac{\ln [d(t) / d(0)]}{t}
$$

Here $d(0)$ - the initial displacement vector phase trajectory, $d(t)$ - shift of the phase trajectory, which occurs over time. The value of $S^{K S}$ is called as the entropy of the Kolmogorov-Sinai or KS-entropy. KS - entropy determined by the dynamic parameter of the system - an exponential Lyapunov instability for Hamiltonian systems.

KS - entropy proved indispensable in the study of problems of dynamic chaos in Hamiltonian systems. For example, using the KS-entropy, we can study regular or chaotic processes [8,9]. But the KS entropy characterizes the system as a whole. It does not answer on the questions, how the system evolves over time to a particular state, depending on the initial and boundary conditions imposed on the system.

In general in the basis submitted here three formulas of entropy there are three different approaches to the analyses of the systems can be selected. The entropy of Clausius is defined entirely within the thermodynamic laws through appropriate thermodynamic parameters. Therefore, the scope of use of this formula entropy is limited by the field of application of thermodynamics. The physical meaning of this entropy determined by the fact that the part of the mechanical work goes into heating the bodies involved in this work.
Boltzmann entropy is built on the principles of probability. Therefore, its scope of use is limited domain of applicability of relevant principles of probability. For example, the ergodic hypothesis has a place. I.e., Boltzmann entropy is applicable in the frames of probabilistic constraints. It can be used for analysis, both equilibrium and nonequilibrium systems. The Gibbs entropy is also belonging to the probabilistic definition of entropy [3]. It is applicable for equilibrium systems. On probabilistic principles are also constructed the Shannon information entropy [8]. The idea that entropy is a measure of chaos and should be his criterion formed the basis for the definition of S-entropy for open nonequilibrium systems [10].

Kolmogorov - Sinai entropy introduced on the basis of invariants of motion, which include the Lyapunov exponents. These indicators determine the general characteristics of almost any systems, although they are difficult to use for the study of the evolution of systems over time.

The above definitions of entropy practically cover all known types, although with each of the existing definitions of entropy has its field of application. But they all share a common limitation. This limitation is that with their help, you cannot answer the question of: how the entropy changes over time in the evolution of systems in accordance with changes in external constraints. They are difficult to use and to analyze the evolution of the system. Moreover, the use of statistical regularities and averaging in (2-3) makes it difficult to study the possibility of actually observed in nature irreversible dynamics in the framework of the laws of mechanics.

In this paper we propose a new formula of entropy, which follows directly from the deterministic motion equations for the system. In contrast to the existing definitions of entropy, it is completely determined by the dynamical parameters of the system and the parameters that determine the external constraints on the system. This formula allows calculating of the entropy of the system at any time without using any statistical hypotheses. Moreover, it allows you to associate the concept of entropy with physical concepts that characterize the dynamics of systems. We will show how this formula of entropy obtained; what are its distinctive features; and how it can be used to study the dynamics of systems.

In this paper we propose a definition of another type of entropy and is given the corresponding formula. This formula follows directly from the motion equation of the structured particles [11]. It follows from this equation due to the fact that one of its members determines the amount of change in internal energy due to the work of external forces. Therefore, it is called dynamic entropy (D-entropy). Unlike 
definition of entropy existing today, the D-entropy is completely determined by the dynamic parameters of the system and the parameters that determine the external constraints on the system. This makes it possible to use this entropy for analyses of the nonequilibrium systems at any time without any statistical hypotheses. Moreover, it is possible to associate the concept of entropy with parameters characterizing the dynamics of systems.

The purpose of this paper is to show how to obtain entropy directly from the dynamical laws without any statistical laws. We will show also the essence of D entropy, what are its distinctive features, and how Dentropy can be used in the study of the dynamics of systems and their statistical properties.

\section{Dynamical entropy}

Determination of dynamic entropy (D-entropy) as the relative change in the internal energy of the system under the action of external inhomogeneous forces was submitted in the mechanics of structured particles (SP) [11, 12]. SP - is equilibrium system from a sufficiently large number of potentially interacting material points (MP). Let us explain what is it the mechanics of SP and how D-entropy appearing in mechanics.

Classical mechanics is built on the basis of bodies models as a set of MP. The motion equations of MP are determined only by the symmetry of space [13].

Mechanics of SP was constructed based on the bodies' models as a set of SP. The SP motion equation was built based on the principle of duality symmetries. The essence of the principle of duality symmetries is that the motion of the system determined by two types of symmetry: by the symmetry of the space in which the system moves and the internal symmetries of the system. The system motion equation was obtained from the dual expression of energy which defined as a sum of two energies: the motion energy of the system and its internal energy [14]. The energy is represented in the micro and macro variables. The internal energy is given in micro-variables which are the coordinates and velocity of the center of mass the system. The motion energy is given in macro variables that determine the motion of the system. Micro and macro variables form two groups of independent variables: the translation group, and a group of variables that determine the motion of MP relative to the center of mass of the system [15]. Consequently, the translation group determines the motion of the system as a whole and micro-variables determine group of chaotic motions MP into SP. When the system moves in an inhomogeneous field of external forces, the micro macro variables are interrelated. Arising symmetry breaking each of these groups of symmetry is manifested in the mutual transformation of two types of energy. It should be emphasized that in the micro and macro variables the symmetry breaking is described by bilinear terms in the expansion of external forces. These forces depend both on the micro and macro variables.

Thus, the fundamental difference between classical mechanics and mechanic of SP is that classical mechanics is based on the symmetry of the only space. Therefore in the frame of the classical mechanics it is impossible describe the violation of invariance of the motion energy.

In mechanics SP the invariant is the sum of the motion energy and internal energy. Therefore mechanics SP allows describe the processes of dissipation associated with the violation of the invariance of the motion energy due to its transformation into internal energy of SP. It is possible when SP moving in a nonuniform field of external forces. The SP mechanics constructed on the basis of the SP motion equation. This motion equation has the form $[11,12]$ :

$$
M_{N} \dot{\vec{V}}_{N}=-\vec{F}^{e n v}-\left(\Phi^{e n v}+\dot{E}_{N}^{i v}\right) \vec{V}_{N} / V_{N}^{2}
$$

Here $\vec{V}_{N}=\bar{R}_{N}=(1 / N) \sum_{i=1}^{N} \dot{\vec{r}}_{i}$ - is a velocity of the center of mass, $i=1,2,3 \ldots N$ - is a number of MP, $M_{N}=N m ; F^{e n v}=\sum F_{i}^{e n v}\left(R_{N}, \tilde{r}_{i}\right), \dot{E}_{N}^{i v s}=\sum_{i=1}^{N} \tilde{v}_{i}\left(m \dot{\widetilde{v}}_{i}+F\left(\tilde{r}_{i}\right)_{i}\right)$ - is a change of the internal energy, $\Phi^{e n v}=\sum \tilde{v}_{i} F_{i}^{e n v}\left(R_{N}, \tilde{r}_{i}\right), \vec{F}_{i}^{e n v}\left(R_{N}, \tilde{r}_{i}\right)$ - is a force which acted on $i$-s MP from the external field of force, $r_{i}=R_{N}+\tilde{r}_{i}, \tilde{r}_{i}$ - is a coordinate of MP relative to the center of mass.

Feature of equation (4) is that it was obtained not the traditional way from the Lagrange equations but by differentiating the energy of SP which represented as the sum of the internal energy of the SP and its motion energy [11]. Such a way of obtaining the equation helped avoid the use of the hypothesis of holonomic constraints [13]. This hypothesis excludes in the Lagrange equations the nonlinear terms which are responsible for the equilibration [14]. Therefore the Lagrange equation is reversible.

Violation of symmetry in each of the two groups' of symmetry of SP when it moves in a nonuniform field of external forces, can be studied by decomposition 
of the right-hand side of eq. (4) with respect to $R^{s} / R^{i n v} \quad$ when $R^{s} \square R^{i n v}$. Here, $R^{s}$ is the characteristic scale of the system, $R^{i n v}$ - is the characteristic scale of the inhomogeneity of the external field of force. From here can be seen that the violation of the invariance of the motion energy and the internal energy are determined by bilinear terms in the expansion of external forces, which depend from the micro and macro variables. The rate of increase of internal energy for SP is proportional to the gradient of the external forces [12].

Irreversibility of processes in the system is connected with the impossibility of transformation of internal energy into kinetic energy of SP. If in the eq. (4) by the standard way go to the thermodynamic variables [1], it is possible to come to the first law of thermodynamics $[11,14]$.

All bodies in nature in general case are the nonequilibrium systems. In the approximation of the local thermodynamic equilibrium, the nonequilibrium systems can be submitted as a set of SP which are in the relative motion to each other $[1,3]$. Then the change in the internal energy of a nonequilibrium system can be determined by the motion equations of $\mathrm{SP}$, as the total amount of change in internal energies of all SP at the expense of the energy of their relative motion.

In a closed nonequilibrium system the external field with respect to each SP is a field of forces from the other SP. According to the eq. (4), the energy of relative motion of SP is decreased due to the work of these forces. By reducing of the relative motion of SP the nonequilibrium system equilibrates. The equilibrium state of the system is determined by the absent of the relative motion of SP [3]. Thus the motion of the system to the equilibrium is determined by the ratio of the amount of increasing of the internal energy of SP to the total value of their internal energies. This ratio we called by the Dentropy, i.e. D-entropy directly follows from the motion equations. The D-entropy can be written as follows [11, 12]:

$$
\Delta S^{d}=\sum_{L=1}^{R}\left\{N_{L} \sum_{k=1}^{N_{L}}\left[\int \sum_{s} F_{k s}^{L} v_{k} d t\right] / E_{L}\right\}
$$

$E_{L}$ - is internal energy $L-\mathrm{SP} ; N_{L^{-}}$is a number of MP in $L$-SP; $L=1,2,3 \ldots R$ - is a number $\mathrm{SP}$ in the nonequilibrium system; $S$ - is external MP which interacted with c $k$-s MP from $L-\mathrm{SP} ; F_{k s}^{L}$-is a component of the force that changes of the velocity of the $k$-s MP relative to the center of mass of $L$ SP. This force acted from the side of $S$ - MP another SP; $v_{k}$-is a velocity of $k$-s MP relative to the center of mass SP.

The eq. (5) defines the relative change in the internal energy of all SP including in the non-equilibrium system due to the work of the forces acting between them. That is, this formula determines what part of the energy of SP relative motion goes into their internal energy. As a result of reducing of the relative motion of energy of SP due to its transformation into internal energy of SP, the nonequilibrium system moves toward equilibrium. Equilibrium occurs when the energy of the relative motion of SP transformed into their internal energy [3]. Because the entropy (5) is determined by the laws of classical mechanics by means of the equations of motion of SP, it is called as dynamical entropy.

Thus, D - entropy is defined by the nonlinear terms of the external forces which depend from the micro and macrovariables. Symmetry breaking occurs under condition of preservation the sum of the motion energies and the internal energies of the system. Irreversibility of processes in the system is related to the impossibility of transformation of internal energy into kinetic energy of SP.

For equilibrium system and, hence, for sufficiently large systems, the D-entropy corresponds to thermodynamic entropy $S^{c}$ (see eq. (1)). This is due to the fact that the only for equilibrium systems, the internal energy equivalent to the thermal energy [1]. And the fact that internal energy only increases due to the motion energy, corresponds to the second law of thermodynamics.

The D- entropy has great versatility, since it is determined by the motion equations of systems which derive from deterministic laws of classical mechanics. Thus it can be used for analyze both equilibrium and nonequilibrium systems. Moreover, since D-entropy is followed from the laws of classical mechanics, D-entropy can in principle be used for determine the region of applications of the thermodynamical laws, as well as for the region of applications of the probabilistic entropies.

So, D - the entropy associated with the change in the internal energy of the system when the system motion in inhomogeneous space. It is not difficult to make sure that the D - entropy is in agreement with all three of the above definitions of entropy, as well as in full compliance with the statistical nature of the equilibration $[1,3]$.

\section{The possibility of using the D-entropy for analysis of dynamical systems}

One of the most important questions in physics is the question of about the limits of validity of the thermodynamic description of systems $[16,17]$. Dentropy allows us to answer this question. 
We are performed the numerical calculations of the change of D-entropy for the system of the potentially interacting MP which moves in an inhomogeneous field of external forces. The calculations were based on the law of conservation of energy of systems. According to this law the sum of the motion energy and the internal energy is invariant of the motion when each of the components of the energy may vary.

The numerical calculations of the change of the internal energy of the system were carried out for the passage of the system with the different number of MP through the potential barrier. The dependence of the change of the internal energy of the systems from the number of MP was studied. The calculations were carried 400 times for a given number of particles for different initial states of the system, but for the same predetermined amount of energy. This made it possible to determine the change of the fluctuations of the internal energy for different states of the system for a given value of its energy of motion and a given number of MP. As a result, it was found that D - entropy for a system of small amounts of MP can be both positive and negative $[18,19]$. This means, for example, that the internal energy of a system from several particles can be transformed into kinetic energy of the system and return.

It was found also that the fluctuations of internal energy decreasing with increasing number of particles in the system for different initial conditions. When $N \leq 64$, the $\mathrm{D}$-entropy can be as positive as negative. But when $N \geq 64$, the internal energy can only increase. I.e., when $N \geq 64$ then none of the 400 numerical experiments gave a negative value change of the internal energy. This means that when $N \geq 10^{2}$ the dynamics of the system becomes irreversible. Therefore the number $N \geq 10^{2}$ can be called as a first critical number of the system, beyond which the system becomes irreversible. Obviously, in the general case, this critical number will depend on the parameters of the task, for example, the width and height of the barrier.

When $N \geq 10^{3}$, the dispersion of the internal energy reaches to the minimum. With further increase in the number of MP the increment of the internal energy is not changed. This number can be called as a second critical number. Thus if in the system consist from $N \geq 10^{3}$, the thermodynamic description is a correct.

The results of numerical calculations of the amplitude fluctuations of the internal energy of the systems are convincing proof of the possibility of justification of statistical laws, based on the D-entropy. In according with these calculations, the amplitude of the fluctuations arising when the system passage from the barrier, are dependent from the number of MP and from distribution function. It turned out that the behavior of these fluctuations corresponds to the law of statistical fluctuations of the mean square value of [3].

Let us recall how on the basis of statistical regularities we were proved that the relative fluctuation of any additive parameter of the system is inversely proportional to $\sqrt{R}$ [3].

The internal energy $E^{\text {ins }}$ of the system is additive value. Therefore, if the system is divided into $R$ subsystems, the average value of its internal energy is equal to the sum of the mean energy values of the internal subsystems. I.e., $\left|E^{i n s}\right|=\sum_{i=1}^{R}\left|E_{i}\right|$, where $E_{i}$ - is energy $i$-s subsystem. We shall start from fact that the internal energy is increases in proportion to the number of MP systems. Then the mean square value of the fluctuations of the internal energy will be equal to: . If the fluctuations in the subsystems considered independent then we get: $\left|(\Delta E)^{2}\right|=\sum_{i=1}^{R}\left|\left(\Delta E_{i}\right)^{2}\right|$. Hence we arrive at the well-known law: $\left|(\Delta E)^{2}\right|^{1 / 2}|| 1 / R^{1 / 2}[3]$.

Thus, if the calculated value of the relative fluctuations $E^{\text {int }}$ that occur when the system passes through the barrier, with the number of MP varies inversely to $\sqrt{R}$, it will serve as proof of the law of the fluctuations and the confirmation of the possibility of the study of law with the help of the laws of classical mechanics. It was found that the approximating dotted line given by the equation: $f=p+r / \sqrt{R}$, where $p=3.5, \quad r=110$ corresponds to the calculated points.

All this means that, firstly, the numerical calculations of the passage of the system through the barrier are true; secondly, the need to submit the energy of the system as a sum of motion energy and the internal energy is displayed in statistical laws; thirdly, the laws of classical mechanics is applicable for substantiate of statistical laws and can be used for determine the scope of their application.

Thus, the D - entropy is useful to determine the range of applicability of statistical and thermodynamical laws for different dynamical tasks.

\section{Conclusion}

The states of any systems, their changes are determined by the dynamic processes within the systems and processes of their interaction with the outside. Thus, the dynamics of the system is 
completely determined by two scalar functions: internal energy and the energy of its motion. The amount of internal energy and the motion energy is a constant when each of these energies can change. This is the law of energy conservation for the open system.

The dynamical processes in the system are described by the micro and macro variables. Internal states within the system defined by the internal energy which is given in micro variables that determine the motion of elements of the system with respect to its center of mass. The dynamics of the system as a whole is determined by the macrovariables. The macrovariables are determined the motion energy of the system in space.

The nonlinear mutual transformation of the motion energy and the internal energy for the moving bodies in an inhomogeneous space are possible. These transformations determine the evolution of the system. One of the important quantity that characterizes this transformation, we have called D entropy. Only in the coordinate system of the dual representation of energy as the sum of the internal energy and the energy of motion with the help of micro and macro variables, this transformation is determined by bilinear terms in the expansion of the field of external forces. The bilinear terms are dependent from micro and macro variables. Therefore, this coordinate system is logical to call "natural coordinate system" describing the dynamics of systems. The very existence of thermodynamics, in particular, the first law of thermodynamics, is the basis argument for selecting the natural coordinate of system, which defines by the principles of duality symmetries, under which the energy acquires of the dual type.

D-entropy is defined as the ratio of the increment of internal energy of the system to the magnitude of this energy. It is expressed in terms of micro and macro variables. Therefore, it is a dynamic expression of entropy. The value of D-entropy is determined by summing the works of external forces, which change of the internal energy. The D-entropy is deterministic since it is uniquely determined by the system motion equation. For equilibrium systems D-entropy up to a numerical factor is equal to the entropy of Clausius. In this case the D-entropy can be expressed in terms of temperature and function of heat. For small systems, the D-entropy, in contrast to the Clausius entropy, can be both positive and negative.

Derivation of the D-entropy is based on a deterministic motion equation of the system. The numerical calculation of the D-entropy, simplifies the determination the region of application of the laws of probability used to describe the evolution of systems, and can be used for determination of the criteria of the applicability of the different statistical methods describing systems. This is confirmed by the numerical calculations of the change in internal energy systems in a heterogeneous field of external forces. Calculations, in particular, showed how the Dentropy can be used to determine the limits of applicability of the thermodynamic description of the system, as well as the limits of applicability of probabilistic statistical laws.

The D-entropy reveals the dynamic nature of the concept of entropy. For example, the second law of thermodynamics is connected with the impossibility of transforming of the internal motion energy of the equilibrium system into its energy of organized motions in according with the D- entropy and the system's motion equation. At the same time, the energy of the relative motion of interacting systems can be transformed into internal energy. The transition of chaotic motion into energy of organized motion is prohibited by law of conservation impulse that follows from the principle of Galileo. This is the essence of the irreversibility and the essence of the nature of the second law of thermodynamics.

Thus to reveal the nature of the entropy, it is necessary to take into account that the bodies' dynamics governed by two types of symmetry: internal symmetry and external symmetry of space. Violation of each of this symmetry is determined by the D-entropy.

\section{Reference}

1. Rumer Yu.B., Rivkin M.Sh. Thermodynamics, Stat. physics and Kinematics. Moscow. Nauka. 1977. 532 p;

2. Lebowitz J.L. Boltzmann's entropy and time's arrow. Phys. Today. 1999. p. 32-38; http://dx.doi.org/10.1063/1.881363

3. Landau L.D., Lifshitz E.M. Statistical physics. Moscow.1976.583 p;

4. Zaslavsky G.M. Stochasticity of dynamic systems. Moscow. Nauka.1984, 273p;

5. Ginzburg V.L. Special session of Editorial board of the Journal of Physics-Uspekhi, honoring the 90th anniversary of VL Ginzburg. Advance Physics of Sci. 2007. 177 (4). p. $345-$ 346;

6. Sinai Y.G. Modern problems of ergodic theory. M.: FIZMATLIT. 1995. 208 p;

7. Sinai Ya. G. Introduction to Ergodic Theory, Princeton University Press, Princeton. New Jersey USA. 1976. 144 p

8. Loskutov A.Y., Mikhailov A.S. Introduction to Synergetics. Nauka, Moscow.1990. 272 p;

9. Loskutov A.Y. The charm of chaos. Phys. 2010.V.150. № 12. p. 1305-1329;

10. Klimontovich Y.L. The statistical theory of open systems. Moscow. Janus. $1995 . \quad 292 \quad$ p; http://dx.doi.org/10.1007/978-94-011-0175-2

11. Somsikov V.M. Thermodynamics and classical mechanics, Journal of physics: Conference series. 23. 2005. p.7-16 http://dx.doi.org/10.1088/1742-6596/23/1/002

12. Somsikov V.M. From the Newton's mechanics to the physics of the evolution. Almaty. 2014. 272 p;

13. Goldstein G. Classical Mechanics. Moscow.1975. 416 p;

14. Somsikov V.M. Why It Is Necessary to Construct the Mechanics of Structured Particles and How to do it. Open Access Library Journal, 2014, 1PP. 1-8, DOI: 10.4236/oalib.1100586; http://dx.doi.org/10.4236/oalib.1100586 
15. Lyubarskii G.Y. Group theory and its applications in physics, Nauka, Moscow 1958,p.354;

16. Smoluchowski M. Boundaries of validity of the second law of thermodynamics. Uspehi Fizicheskih Nauk. 1967. v.93. iss. 4. p. 724-737;

17. Haytun S.D. Mechanics and irreversibility. (1996). Janus. Moscow. 448 p;

18. Somsikov V.M., Denisenya M.I. Features of the oscillator passing through potential barrier. Proceedings of the universities. Series Physics. №. 3. March. 2013. p. 95-103 http://dx.doi.org/10.1007/s11182-013-0056-y

19. Somsikov V.M., Andreyev A.B., Mokhnatkin A.I. Relation between classical mechanics and physics of condensed medium International Journal of Physical Sciences. Vol. 10(3),

pp.

112-122. 